\title{
KIC 4247791: a SB4 system with two eclipsing binaries (2EBs) (Research Note)
}

\section{A quadruple system?^}

\author{
H. Lehmann ${ }^{1}$, M. Zechmeister ${ }^{2}$, S. Dreizler ${ }^{2}$, S. Schuh ${ }^{2}$, and R. Kanzler ${ }^{2}$ \\ 1 Thüringer Landessternwarte Tautenburg (TLS), Sternwarte 5, 07778 Tautenburg, Germany \\ e-mail: lehm@tls-tautenburg. de \\ 2 Georg-August-Universität, Institut für Astrophysik, Friedrich-Hund-Platz 1, 37077 Göttingen, Germany \\ e-mail: [zechmeister;dreizler; schuh;rkanzler]@astro.physik.uni-goettingen.de \\ Received 19 October 2011 / Accepted 14 February 2012
}

\section{ABSTRACT}

\begin{abstract}
Context. KIC 4247791 is an eclipsing binary observed by the Kepler satellite mission.
Aims. We wish to determine the nature of its components and in particular the origin of a shallow dip in its Kepler light curve that previous investigations have been unable to explain in a unique way.

Methods. We analyse newly obtained high-resolution spectra of the star using synthetic spectra based on atmosphere models, derive the radial velocities of the stellar components from cross-correlation with a synthetic template, and calculate the orbital solution. We use the JKTEBOP program to model the Kepler light curve of KIC 4247791.

Results. We find KIC 4247791 to be a SB4 star. The radial velocity variations of its four components can be explained by two separate eclipsing binaries. In contradiction to previous photometric findings, we show that the observed composite spectrum as well as the derived masses of all four of its components correspond to spectral type F.

Conclusions. The observed small dip in the light curve is not caused by a transit-like phenomenon but by the eclipses of the second binary system. We find evidence that KIC 4247791 might belong to the very rare hierarchical SB4 systems with two eclipsing binaries.
\end{abstract}

Key words. binaries: eclipsing - binaries: spectroscopic - stars: fundamental parameters

\section{Introduction}

The Kepler mission was designed and launched with the primary aim of searching for transiting planets, and indeed, has found hundreds of planetary candidates (Borucki et al. 2011) and a large number of eclipsing binaries (EBs). In addition to those already very interesting objects, even more unusual systems such as multiple transiting planets (Lissauer et al. 2011) or triply eclipsing triples (Carter et al. 2011; Derekas et al. 2011; Slawson et al. 2011) have been discovered.

In their analysis of low-mass EBs in the Kepler Q0 and Q1 data releases, Coughlin et al. (2011), hereafter C11, find that one object does not belong to the main-sequence, KIC $4247791=2$ MASS J 19083956+3922369. The authors determine its temperature to be $T_{\text {eff }}=4063 \mathrm{~K}$, an orbital period of 4.100866 , a combined mass of $1.28 M_{\odot}$, and a combined radius of $3.82 R_{\odot}$. According to these values, the system must contain at least one evolved component. Moreover, a periodic transit-like feature is superimposed on the EB light curve. With respect to the main eclipse, this feature drifts with time (Fig. 1) and the authors derive possible periods of either 2.02484 or 4.04969. According to C11, the feature could be explained in the case of the shorter period by (1) a background EB with no visible secondary; (2) a circumbinary transiting object; or (3) a transiting object around one of the stars in an almost 2:1 resonant orbit with the binary. In the case of the longer period, the explanation could be (4) a fore- or background EB with 0.98752 times

* Based on observations with the 2-m Alfred-Jensch-Telescope of the Thüringer Landessternwarte Tautenburg.

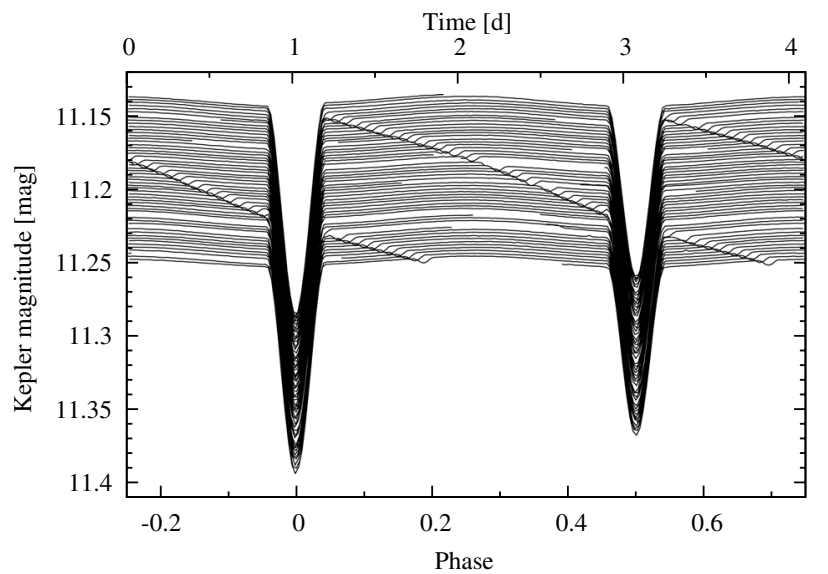

Fig. 1. Detrended Kepler light curve (Q0-Q3 data) phase folded to 4. 100871. A shallow dip drifts with time. Around 55 cycles are covered, each shifted upwards by 2 mmag (times the cycle number) for visibility.

the main period of KIC 4247791 that has nearly identical depths of the primary and secondary eclipses.

KIC 4247791 also has an entry in the Kepler eclipsing binary catalogue (Prša et al. 2011) and both EBs are included in its second release (Slawson et al. 2011).

We obtained 15 high-resolution spectra covering the orbital period of KIC 4247791 well and use them in this investigation to find the real cause of the observed transit-like feature in the Kepler light curve of the star and to derive tighter constraints on the fundamental parameters of its components. For this purpose, we measure the radial velocities (RVs) of the components of 


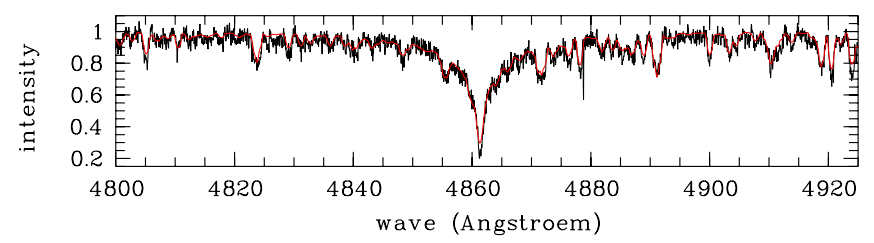

Fig. 2. Observed spectrum in the $\mathrm{H} \beta$ region (black, $\mathrm{BJD}=2455819$ ) and best-fit synthetic spectrum (red) computed for $T_{\text {eff }}=6400 \mathrm{~K}$, $\log g=3.7,[\mathrm{M} / \mathrm{H}]=-0.2 \mathrm{dex}$, and $v \sin i=40 \mathrm{~km} \mathrm{~s}^{-1}$.

KIC 4247791, analyse the averaged composite spectrum, derive the orbits of the found binary systems, and combine the results with those obtained from the Kepler light curve analysis.

\section{Observations}

All spectra have been taken with the Coude-Echelle spectrograph attached to the 2-m Alfred-Jensch-Telescope of the Thüringer Landessternwarte Tautenburg. The spectra cover the wavelength range $4700 \AA$ to $7400 \AA$ with a resolution of 32000 . The exposure time was 40 min per spectrum. The dates of observation are listed in Table A.1. Spectrum reduction was done using standard ESO-MIDAS packages including bias and straylight subtraction, filtering of cosmic rays, flat-fielding using a halogen lamp, optimal order extraction, wavelength calibration using a ThAr lamp, and normalization to the local continuum. Nightly shifts in the instrumental zero point were corrected by using a large number of telluric $\mathrm{O}_{2}$ lines.

\section{Spectrum analysis}

In a first step, we determined the predominant spectral type from one observed, composite spectrum showing the sharpest (unsplit) lines. For the determination of the stellar parameters, we used a method identical to that described in Lehmann et al. (2011), to derive values of $T_{\text {eff }}=6400 \mathrm{~K}, \log g=3.7$, $v \sin i=40 \mathrm{~km} \mathrm{~s}^{-1}$, and a metallicity of -0.2 dex relative to the solar value. Figure 2 compares an observed spectrum with the best-fitting synthetic spectrum for the $\mathrm{H} \beta$ region. The results are representative of the composite spectrum. This spectrum has to be decomposed to derive the spectral types of the single components. The derived temperature corresponds to spectral type $\mathrm{F}$ and contradicts the value of $4063 \mathrm{~K}$ given by $\mathrm{C} 11$. In particular, the observed Balmer line profiles are incompatible with such a low temperature value.

\section{Orbital solutions from cross-correlation}

Cross-correlation functions (CCFs) were obtained from the cross-correlation of the observed spectra with the synthetic template spectrum derived in Sect. 3 but using $v \sin i=1 \mathrm{~km} \mathrm{~s}^{-1}$ instead of $40 \mathrm{~km} \mathrm{~s}^{-1}$ to obtain the maximum spectral resolution in the CCFs. In most of the CCFs, we discovered four components. The positions of these components represent the RVs of four stars in two binary systems with orbital periods close to the periods of 4.100866 (the two stronger peaks in the CCFs) and 4.049687 (the two smaller peaks) given in C11. This can be seen clearly if we arrange the CCFs according to the positions in orbital phase based on the two periods (Fig. 3).

The line splitting can also be seen in the single spectra. Figure 4 compares the Fe I $4957 \AA$ line from two spectra taken at different orbital phases where the noise was reduced by averaging over 9 wavelength bins. One spectrum clearly shows a splitting into four components belonging to system I, its stars A and B, and system II, its stars A and B, whereas the other spectrum has almost no splitting.

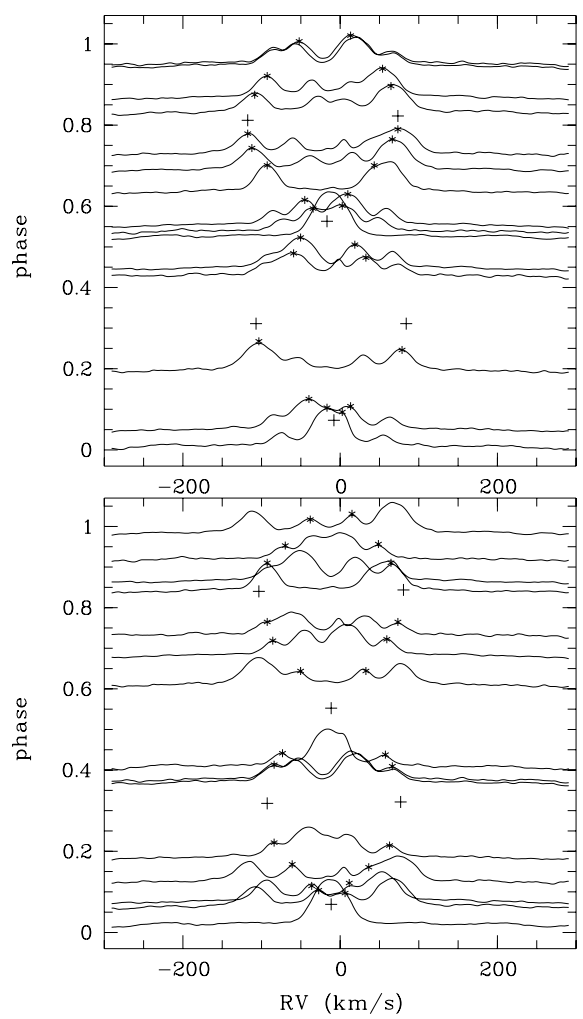

Fig. 3. CCFs vertically shifted with orbital phase based on periods of 4.100866 (top) and 4.049687 (bottom). The peak positions belonging to the corresponding orbit are marked by asterisks, and the positions of minimum and maximum RV separation by plus signs.

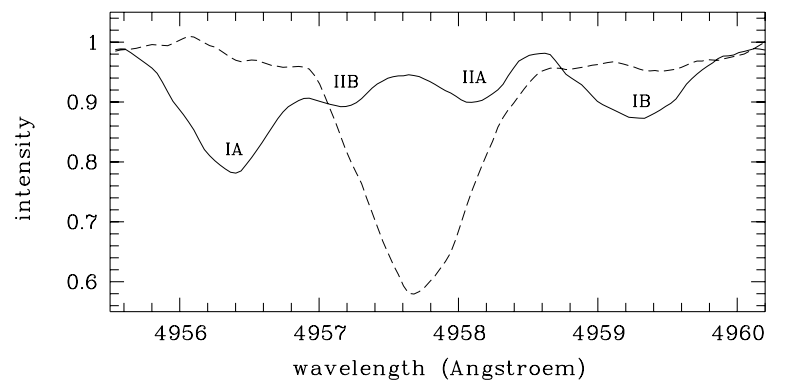

Fig. 4. The Fe I $4957 \AA$ line observed at two different orbital phases showing strong (continuous line) and almost no (dashed line) splitting.

The RVs were determined from the CCFs using multiple Gaussian fits. From 14 spectra, we could determine the RVs of all components. We used four Gaussians in 12 cases and five Gaussians in the 2 cases where we detected a fifth contribution produced by weak telluric lines. In one spectrum, we could only resolve the RVs of the brighter EB. Table A.1 lists the RV values obtained for the four components.

The orbital solutions were calculated using the method of differential corrections to the orbital elements (Sterne 1941). We assumed circular orbits in each case. For both systems, we fixed the orbital periods and times of $\gamma$-velocity passage/primary minimum (iterated with those obtained in Sect. 5). Figure 5 shows the RVs of both systems and the corresponding $\mathrm{O}-\mathrm{C}$ values folded with the two different periods. Three outliers can be seen for component IA, one for IB and one for IIB. These outliers were rejected when calculating the orbital solutions. They can arise when the RV components coincide and are poorly resolved in the CCFs, in particular at/around phases 0 and 0.5 , which moreover can be affected by the Rossiter effect (for A 
H. Lehmann et al.: KIC 4247791: a SB4 system with two eclipsing binaries (2EBs) $(R N)$
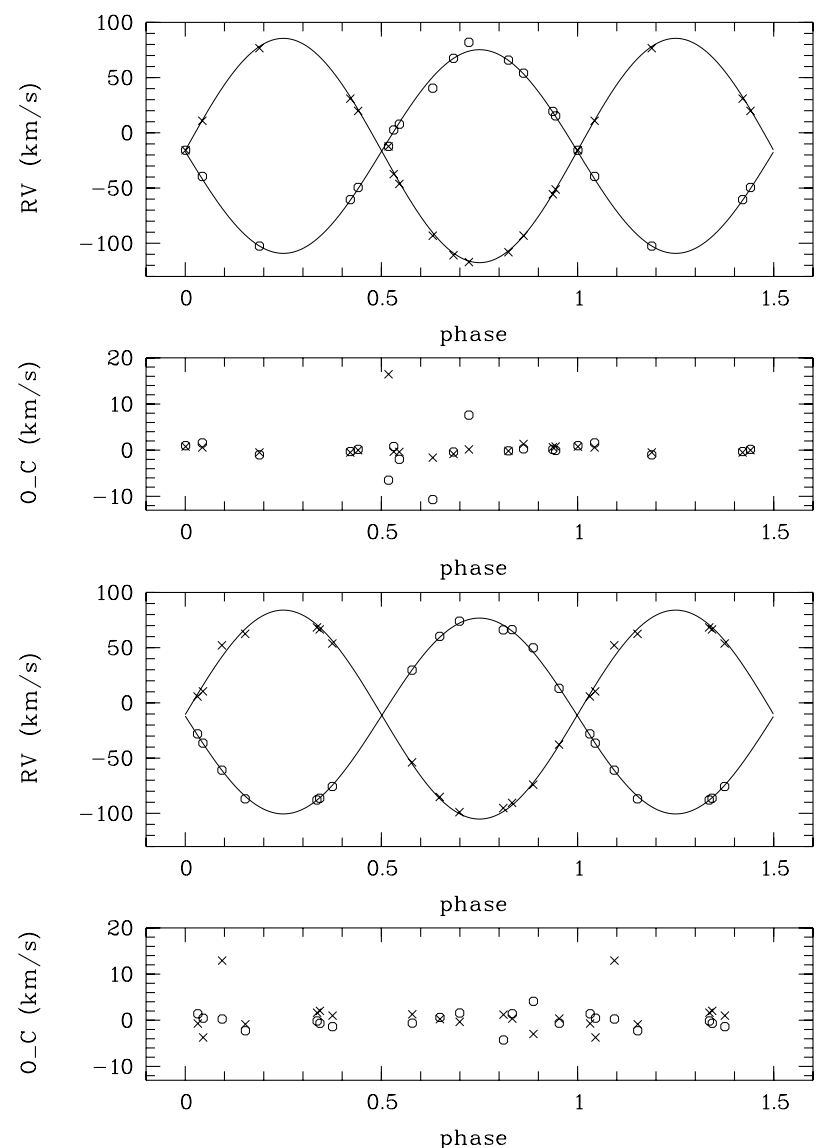

Fig. 5. From top to bottom: RVs of components IA (circles) and IB (crosses) folded with the period of $4.100871, \mathrm{O}-\mathrm{C}$ residuals of IA and IB, RVs of components IIA (circles) and IIB (crosses) folded with the period of 4.049732, and O-C residuals of IIA and IIB.

and $\mathrm{B}$, respectively). The derived orbital elements are listed in the upper part of Table 1.

Phase zero in Fig. 5 corresponds to the time of primary minimum ( $T_{\mathrm{MIN}}$ in Table 1$)$. We see that in both systems the RVs of the more massive components A that have slightly smaller RV amplitudes, decrease at this phase. This means that the more massive components are eclipsed at $T_{\mathrm{MIN}}$ and should have higher surface brightnesses. It is remarkable that not only the periods of the two systems are almost identical (but significantly different as can also be seen from their Kepler light curves) but that also the RV semi-amplitudes $K$ and the mass ratios $q$ are very similar.

\section{Light curve analysis}

The Kepler database provides publicly available data for 228 days (2009-05-02 to 2009-12-16; quarters Q0-Q3) of photometric observations of KIC 4247791 (Fig. 1), including 88 days taken in short cadence mode (SC, Q2, 1 min exposure time). On the basis of JKTEBOP (Southworth et al. 2004a,b), the light curve was analysed simultaneously for both EBs using the IDL implementation mpfit of the Levenberg-Marquardt algorithm. A fit was made to the two binary light curves using 14 free parameters in each case: binary period, phase zero-point, ratio and sum of the radii, inclination, surface brightness ratio, eccentricity $(e \sin \omega, e \cos \omega)$, quadratic limb-darkening, and reflection effect coefficients. The mass ratio was fixed to the values determined from the RV measurements. The relative brightness ratio as well as a contribution from a (constant) fifth background light was also taken into account.
Table 1. Parameters and spectral types $(\mathrm{SpT})$ derived from the spectroscopic, light curve, and combined analysis.

\begin{tabular}{lcccc}
\hline \hline & \multicolumn{2}{c}{ System I } & \multicolumn{2}{c}{ System II } \\
& $\mathrm{A}$ & $\mathrm{B}$ & $\mathrm{A}$ & $\mathrm{B}$ \\
\hline$K\left[\mathrm{~km} \mathrm{~s}^{-1}\right]$ & $91.9(4)$ & $101.6(3)$ & $88.6(3)$ & $91.4(4)$ \\
$\gamma\left[\mathrm{km} \mathrm{s}^{-1}\right]$ & $-16.2(9)$ & $-17.1(8)$ & $-11.8(2.0)$ & $-11.7(1.7)$ \\
$q$ & $0.905(7)$ & \multicolumn{2}{c}{$0.970(7)$} \\
\hline$P[\mathrm{~d}]$ & $4.100871(4)$ & \multicolumn{2}{c}{$4.049732(71)$} \\
$T_{\mathrm{MIN}}[\mathrm{BJD}]$ & \multicolumn{2}{c}{$1.1453(1)$} & \multicolumn{2}{c}{$1.353(1)$} \\
$i[\mathrm{deg}]$ & \multicolumn{2}{c}{$79.5(1)$} & \multicolumn{2}{c}{$80.0(8)$} \\
$e \cos \omega$ & $0.00012(6)$ & \multicolumn{2}{c}{$0.0020(3)$} \\
$e \sin \omega$ & $-0.00570(4)$ & \multicolumn{2}{c}{$0.0009(4)$} \\
$e$ & $0.00570(4)$ & \multicolumn{2}{c}{$0.0022(3)$} \\
$\omega[\mathrm{deg}]$ & \multicolumn{2}{c}{$178.7(6)$} & $65(24)$ \\
$R / a$ & $0.1570(4)$ & $0.1503(4)$ & $0.100(9)$ & $0.092(8)$ \\
$L / L_{\mathrm{AB}}$ & $0.576(1)$ & $0.424(1)$ & $0.55(1)$ & $0.45(1)$ \\
$L_{\mathrm{AB}} / L_{\mathrm{I}+\mathrm{II}}$ & \multicolumn{2}{c}{$0.76(2)$} & \multicolumn{2}{c}{$0.24(1)$} \\
\hline$a[\mathrm{AU}]$ & \multicolumn{2}{c}{$0.0742(2)$} & \multicolumn{2}{c}{$0.0681(2)$} \\
$R\left[R_{\odot}\right]$ & $2.50(1)$ & $2.40(1)$ & $1.46(13)$ & $1.35(12)$ \\
$M_{\mathrm{AB}}\left[M_{\odot}\right]$ & \multicolumn{2}{c}{$3.24(3)$} & \multicolumn{2}{c}{$2.56(3)$} \\
$M\left[M_{\odot}\right]$ & $1.70(2)$ & $1.54(1)$ & $1.30(2)$ & $1.26(2)$ \\
$\log g[\mathrm{dex}]$ & $3.87(1)$ & $3.87(1)$ & $4.22(8)$ & $4.28(8)$ \\
$\mathrm{SpT}$ & $\mathrm{F} 0 \mathrm{IV}$ & $\mathrm{F} 2 \mathrm{IV}$ & $\mathrm{F} 7 \mathrm{~V}$ & $\mathrm{~F} 8 \mathrm{~V}$ \\
\hline
\end{tabular}

Notes. $T_{\mathrm{MIN}}$ gives BJD-2 455000 , errors in units of the last digits are given in parentheses.

The Kepler light curves are influenced by instrumental effects, which were treated separately for each quarter or even for sections separated by an interruption of the observations. This is fitted simultaneously with the binaries by piecewise second or third order polynomials. Starting from an initial guess for a total of 52 parameters using identical stellar parameters, zero eccentricity, zero fifth light, and neglecting systematic trends, we employed the IDL routine mpfit to perform an optimisation. mpfit calls JKTEBOP twice to evaluate the contribution of each binary. Since several of the parameters are closely related, the result of the optimisation depends partly on the initial conditions. After a 5- $\sigma$ clipping to remove outliers, we therefore started 50 optimisation runs where the initial conditions were randomly varied around the first result. The one with the lowest $\chi 2$ was taken as the final result. mpfit also provides errors from the diagonal of the covariance matrix. For more realistic errors in the presence of correlated noise, we however, followed the approach of Southworth (2008). To take nonGaussian residuals into account, a series of 500 synthetic light curves was constructed using the best-fit model adding arbitrarily shifted residuals as noise. Since the residuals from the long and short cadence data differed significantly, they were treated separately. These light curves were again fitted using various start values. From the nearly Gaussian distribution of the parameters around the best-fit relation, we determined the $1-\sigma$ uncertainty limits. These are typically an order of magnitude larger than the formal errors provided by mpfit.

The derived parameters are listed in Table 1 and Fig. 6 illustrates the quality of the fit. In addition, we verified the results by fitting each EB separately. For this purpose, the data were decomposed iteratively by phase folding the light curve to the periods of the EBs and calculating the medians in oneminute bins. Many cycles had to be be covered to minimise the residual correlation effects introduced by the ellipsoidal variations and the similar periods of both EBs. The Q2-SC data covers about 21 orbit cycles of EB I and $27 \%$ of the full beat cycle (324.5 days) of both EBs. 

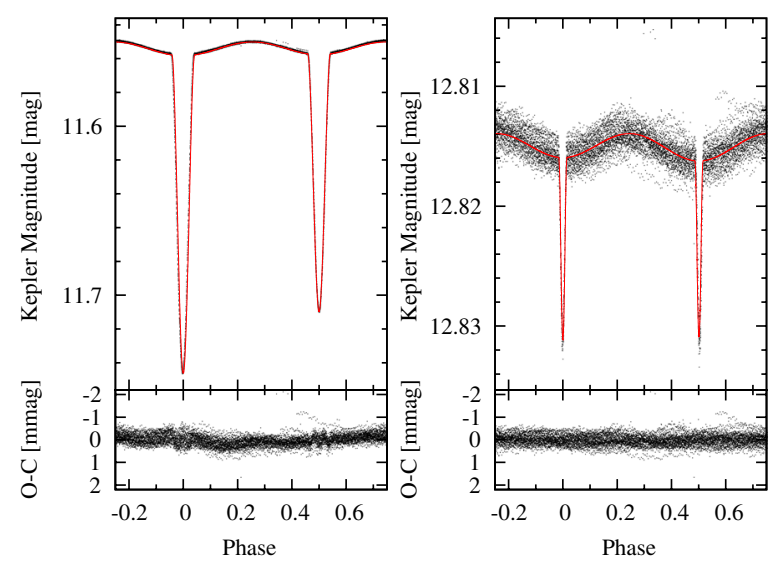

Fig. 6. Disentangled and phase-folded light curves. Left: light curve (EB II model subtracted) fitted by the EB I model. Right: light curve (EB I model subtracted) fitted by the EB II model. The corresponding $\mathrm{O}-\mathrm{C}$ residuals are shown in the lower panels.

With the known periods, inclinations, and RV amplitudes of each component, we were able to derive the stellar radii and masses listed in Table 1. From the masses and radii, we roughly estimated the spectral types using the tables of Gray (2005). We derived a spectral type $F$ for all four stars, which agrees with the observed composite spectrum. For main-sequence stars, we would expect radii of about $1.5 R_{\odot}$ for the stars of system I and of $1.3 R_{\odot}$ for the stars of system II and $\log g$ of about 4.3 for all the components (Gray 2005). Comparing these values with Table 1 , we see that both components of system I must be slightly evolved.

From the derived orbital period and the stellar radii, we were able to estimate the $v \sin i$ of the components of system I assuming synchronized rotation. We measured $31 \mathrm{~km} \mathrm{~s}^{-1}$ for component $\mathrm{A}$ and $30 \mathrm{~km} \mathrm{~s}^{-1}$ for $\mathrm{B}$. This is compatible with the $40 \mathrm{~km} \mathrm{~s}^{-1}$ derived from the composite spectrum in Sect. 3 that we should recall is affected by an additional broadening since this spectrum was taken close to but not exactly during the (double) $\gamma$-velocity passage.

\section{Discussion}

Our results clearly show that the effects observed in the Kepler light curve can be explained by two EBs and not by some transitlike phenomenon. It is difficult to understand, however, that all the similarities in the spectroscopically derived parameters of the two binary systems are caused by chance and one has to ask wether there is a possible connection between the systems. Therefore, the most interesting question is whether both EBs are gravitationally bound and form a quadruple system.

To exclude visible blends, we did a careful search for any contaminants in the Kepler pixel data and in available groundbased images of the corresponding field of view. KIC 4247791 can be found in one $J$-band image of the UKIRT InfraRed Deep Sky Survey (UKIDSS ${ }^{1}$, DR WSERV4, see Lawrence et al. 2007), as well as in the Sloan Digital Sky Survey (SDSS-III ${ }^{2}$, DR8) in all five $(u, g, r, i, z)$ bands. The UKIRT image has the higher spatial resolution with a pixel scale of $0.2 /$ pixel but the image of KIC 4247791 itself is overexposed. The SDSS provides data of 0.'396/pixel and KIC 4247791 is non-saturated in $u$ and $z$, where the Gunn- $z$ image has the better seeing. We used the UKIRT $J$-band image to check for possible blends in the vicinity

\footnotetext{
1 http://surveys.roe.ac.uk/wsa

2 http://wwW.sdss3.org
}

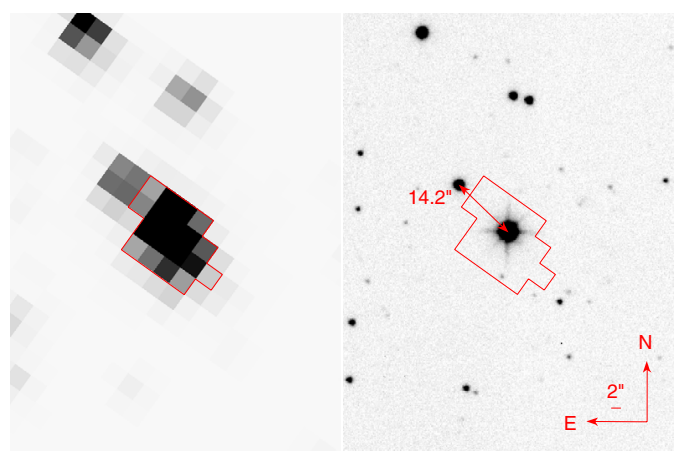

Fig. 7. Left: $1.12^{\prime} \times 1.50^{\prime}$ section of a Kepler full-frame image (FFI). The used extraction aperture for KIC 4247791 is marked by the red borders. Right: UKIRT $J$-band image of the same region. The Kepler aperture is overlaid. The marked, nearby blend is KIC 4247807.
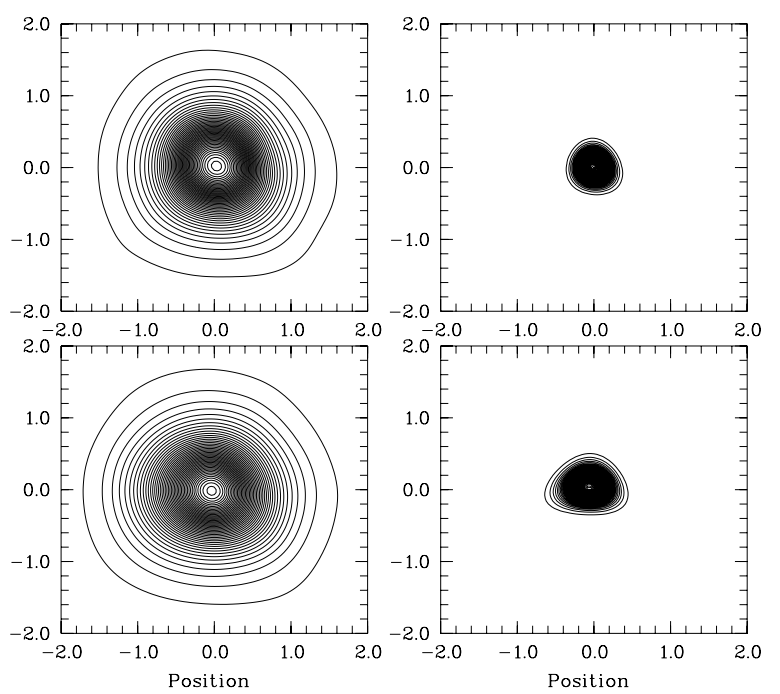

Fig. 8. Top: contour plots of the SDSS z-band image of KIC 4247791 (left) and of the image deconvolved with the PSF (right). The position scale is in arcsec. Bottom: the same with a simulated faint companion in $0 . .5$ distance.

of the star and the SDSS $z$-band image to investigate its flux profile.

An inspection of the $J$-band image and a comparison with the pixel data of the Kepler images identified an object (KIC 4247807, Kepler magnitude $K_{\mathrm{p}}=14.808 \mathrm{mag}$ ) separated by 14 ". 2 from KIC $4247791\left(K_{\mathrm{p}}=11.260 \mathrm{mag}\right)$ close to the aperture used for extraction (Fig. 7). However, the following finding excludes the possibility that KIC 4247807 is EB II: the spectra were taken with a $2^{\prime \prime}$ slit, where the slit orientation on the sky varies with the hour angle of the telescope. For any object that did not lie completely inside the slit, we were unable to derive as precise a RV curve as we obtained from the spectral features of EB II. The close object is 3.5 mag fainter than KIC 4247791. Assuming that $10 \%$ of the light of KIC 4247807 from the PSF wings falls into the aperture, its fifth light contribution should be at a level well below $1 \%$.

Figure 8 shows in its upper left panel a contour plot of the $z$-band image of KIC 4247791. To enhance the resolution, we constructed the mean point spread function (PSF) from all brighter and non-saturated star images in the z-band field by applying a shift and add, sampling all images to ten times higher resolution than provided by the original ones. Two-dimensional Gaussian fits where used to eliminate all subimages showing any asymmetry in the flux profiles. We ended up with a PSF built from 63 star images with a 1'.08 FWHM, the same value as we 
obtained from the image of KIC 4247791 itself. The upper right panel in Fig. 8 shows the image of the star deconvolved with the PSF using the Lucy-Richardson algorithm (Lucy 1974). It is almost perfectly symmetric, possessing a FWHM of 0.'32. For comparison, we simulated a nearby star of 0.2 times the brightness of KIC 4247791 at a distance of 0.'5 using the derived PSF. The result is shown in the lower panels of Fig. 8. The deconvolved image is clearly asymmetric having a FWHM of 0.44 in the direction towards the faint companion and of 0.35 in the perpendicular direction. We conclude that the separation between EB I and EB II must be closer than 0.'5, which still implies that they may be either affected by an accidental blend or be gravitationally bound systems.

There are some findings that are consistent with a quadruple configuration of KIC 4247791 such as the luminosity ratio that provides similar distances of both EBs or the similar inclinations and masses that are indicative of a common formation. The similarity between the systemic RVs also implies that it is a physical associated system. The small difference in the system $\mathrm{RV}$ s of $\sim 5 \mathrm{~km} \mathrm{~s}^{-1}$ could, however, be due to the orbital motion of both EBs around each other and might be used to derive an upper limit to the separation between both EBs. For a circular and edge-on orbit, this upper limit would be 1200 years. We note that this limit is proportional to $\sim K^{-3}$ meaning that if we observe e.g. only a quarter of the maximum RV difference the period would shorten to about 20 years. In the latter case, the corresponding variations in the systemic $\gamma$-velocities of the two systems should be safely detectable from observations spanning about two years.

\section{Conclusions}

Using the period derived in $\mathrm{C} 11$ from the minima in the Kepler light curve, we have been able to model the RV variations of the two main components identified in the CCFs derived from our newly obtained spectra. Two additional features have been observed in almost all the CCFs, which vary with the period of 4.049751 that was proposed by $\mathrm{C} 11$ as a possible period to explain the occurrence of a shallow dip in the Kepler light curve drifting with time against the main minima.

Our spectroscopic analysis has uncovered four stars moving in two separate binary systems. The basic evidence for such an interpretation comes from the time-dependent splitting of the spectral lines into four components that we have been able to assign in a unique way to four stellar components moving in two separate orbits owing to the small difference between the two periods being significant, and visible as small moving dips in the Kepler light curve.

Our results for the combined photometric and spectroscopic observations are consistent with the scenario of two binaries, both lying along the same line of sight, showing eclipses, having almost the same orbital periods, RV amplitudes, and mass ratios. This seems to be a very rare if not unlikely scenario and raises the questions of whether these two systems are physically connected. The observed similarities may be indicative of a quadruple system, although we cannot entirely rule out a moderate back- or foreground scenario.

All derived fundamental parameters of EBI are well defined. For the primary and secondary, respectively, we obtained masses of $1.70 \pm 0.02 M_{\odot}$ and $1.54 \pm 0.01 M_{\odot}$, and radii of $2.50 \pm 0.01 R_{\odot}$ and $2.40 \pm 0.01 R_{\odot}$ and $\log g$ of $3.87 \pm 0.01$ for both components, corresponding to slightly evolved stars of early F-type. For EB II, the derived masses have similar accuracy to that of EBI but the errors in the radii and $\log g$ are distinctly larger. We obtained $\quad M_{\mathrm{A}}=1.30 \pm 0.02 M_{\odot}, \quad R_{\mathrm{A}}=1.46 \pm 0.13 R_{\odot}, \quad$ and $\log g_{\mathrm{A}}=4.22 \pm 0.08$ for the primary and $M_{\mathrm{B}}=1.26 \pm 0.02 M_{\odot}$, $R_{\mathrm{B}}=1.35 \pm 0.12 R_{\odot}, \quad$ and $\log g_{\mathrm{B}}=4.28 \pm 0.08 M_{\odot}$ for the secondary corresponding to late F-type main sequence stars.

To our knowledge, only two stars have been found so far to host two EBs in hierarchical systems, the visual binaries ADS 9537 AB and V994 Her. ADS 9537 AB was discovered by Batten \& Hardie (1965) to consist of two W UMa-type EBs where the two systems orbit each other with a period of about $22000 \mathrm{yr}$ (Batten \& Lu 1986). Lee et al. (2008) discovered that the SB4 star V994 Her hosts two EBs consisting of B8+A0 and A2+A4 main sequence stars. From astrometric data, they estimate a period of the visual binary of few thousand years. There are a few other remarkable objects. Shkolnik et al. (2008) discovered a SB4 quadruple system, BD-22 5866, consisting of one $(\mathrm{K} 7+\mathrm{K} 7) \mathrm{EB}$ and a second $(\mathrm{M} 1+\mathrm{M} 2)$, non-EB system, orbiting each other with a period shorter than $10 \mathrm{yr}$. OGLE 051343.14691837.1 was proposed as a SB4 candidate with two EBs by Ofir (2008) but has since been found to be SB2 (Kołaczkowski et al. 2010; Rivinius et al. 2011). The SB3 quadruple system V379 Cep was found by Harmanec et al. (2007) to host two binaries where one is an EB.

Further observations would be necessary to confirm the quadruple nature of KIC 4247791, namely data extending the present time base and designed to detect the orbital motion between systems I and II based on transit timing or RV variations. In a forthcoming paper, we intend to decompose the spectra of the components using the KOREL program (Hadrava 2006) and we will analyse in greater detail the decomposed light curves and spectra to provide more stringent constraints on the fundamental stellar parameters, in particular the effective temperatures of the components, and hence the evolutionary state and distance modulus of KIC 4247791.

Acknowledgements. This research has made use of SDSS-III. Funding for SDSS-III has been provided by the Alfred P. Sloan Foundation, the Participating Institutions, the National Science Foundation, and the US Department of Energy Office of Science. The SDSS-III web site is http://www.sdss3.org/. MZ acknowledges financial support from the Deutsche Forschungsgemeinschaft (DFG) under RE 1664/4-1.

\section{Appendix A: Measured radial velocities}

Table A.1 lists the RVs measured for the four components of KIC 4247791.

Table A.1. RVs in $\mathrm{km} \mathrm{s}^{-1}$ derived from the four components in the CCFs.

\begin{tabular}{lcccc}
\hline \hline BJD-2455000 & $R V_{\text {IA }}$ & $R V_{\text {IB }}$ & $R V_{\text {IIA }}$ & $R V_{\text {IIB }}$ \\
\hline 700.529717 & 7.82 & -46.25 & 60.25 & -85.15 \\
702.571156 & -39.50 & 10.92 & -86.85 & 62.50 \\
725.484044 & 40.41 & -93.13 & 66.07 & -95.22 \\
726.433284 & 53.99 & -93.06 & -36.43 & 10.44 \\
734.477064 & 65.83 & -108.09 & -28.07 & 5.81 \\
754.407123 & 67.44 & -110.81 & 13.18 & -37.77 \\
757.429167 & -60.46 & 30.93 & 73.98 & -98.91 \\
782.488513 & 2.61 & -37.39 & 49.85 & -74.03 \\
787.374417 & 81.94 & -117.03 & -60.90 & 52.13 \\
788.514326 & -15.77 & -15.77 & -75.66 & 53.74 \\
793.387013 & -102.52 & 76.62 & 29.65 & -53.81 \\
794.419142 & -49.49 & 19.83 & 66.29 & -90.44 \\
796.454312 & 19.35 & -55.60 & -87.92 & 68.29 \\
796.483258 & 15.31 & -51.26 & -86.30 & 66.50 \\
819.341840 & -12.25 & -12.25 & - & - \\
\hline$\triangle R V$ & 0.15 & 0.15 & 0.28 & 0.24 \\
\hline
\end{tabular}

Notes. $\triangle R V$ gives the mean measurement error. 
A\&A 541, A105 (2012)

\section{References}

Batten, A. H., \& Hardie, R. H. 1965, AJ, 70, 666

Batten, A. H., \& Lu, W. 1986, PASP, 98, 92

Borucki, W. J., Koch, D. G., Basri, G., et al. 2011, ApJ, 728, 117

Carter, J. A., Fabrycky, D. C., Ragozzine, D., et al. 2011, Science, 331, 562

Coughlin, J. L., López-Morales, M., Harrison, T. E., Ule, N., \& Hoffman, D. I. 2011, AJ, 141, 78

Derekas, A., Kiss, L. L., Borkovits, T., et al. 2011, Science, 332, 216

Gray, D. F. 2005, The Observation and Analysis of Stellar Photospheres, 3rd edn. (New York: Cambridge University Press)

Hadrava, P. 2006, Ap\&SS, 304, 337

Harmanec, P., Mayer, P., Prša, A., et al. 2007, A\&A, 463, 1061

Kołaczkowski, Z., Mennickent, R., \& Rivinius, T. 2010, in Binaries - Key to Comprehension of the Universe, ed. A. Prša, \& M. Zejda, ASP Conf. Ser., 435,403
Lawrence, A., Warren, S. J., Almaini, O., et al. 2007, MNRAS, 379, 1599 Lee, C.-U., Kim, S.-L., Lee, J. W., et al. 2008, MNRAS, 389, 1630

Lehmann, H., Tkachenko, A., Semaan, T., et al. 2011, A\&A, 526, A124

Lissauer, J. J., Ragozzine, D., Fabrycky, D. C., et al. 2011, ApJS, 197, 8 Lucy, L. B. 1974, AJ, 79, 745

Ofir, A. 2008, Information Bulletin on Variable Stars, 5868, 1

Prša, A., Batalha, N., Slawson, R. W., et al. 2011, AJ, 141, 83

Rivinius, T., Mennickent, R. E., \& Kołaczkowski, Z. 2011, in IAU Symp. 272, ed. C. Neiner, G. Wade, G. Meynet, \& G. Peters, 541

Shkolnik, E., Liu, M. C., Reid, I. N., et al. 2008, ApJ, 682, 1248

Slawson, R. W., Prša, A., Welsh, W. F., et al. 2011, AJ, 142, 160

Southworth, J. 2008, MNRAS, 386, 1644

Southworth, J., Maxted, P. F. L., \& Smalley, B. 2004a, MNRAS, 351, 1277

Southworth, J., Zucker, S., Maxted, P. F. L., \& Smalley, B. 2004b, MNRAS, 355, 986

Sterne, T. E. 1941, Proceedings of the National Academy of Science, 27, 175 